\title{
A Model-based Hierarchical Controller for Legged Systems subject to External Disturbances
}

\author{
Guiyang Xin, Hsiu-Chin Lin, Joshua Smith, Oguzhan Cebe, and Michael Mistry
}

\begin{abstract}
Legged robots have many potential applications in real-world scenarios where the tasks are too dangerous for humans, and compliance is needed to protect the system against external disturbances and impacts. In this paper, we propose a model-based controller for hierarchical tasks of legged systems subject to external disturbance. The control framework is based on projected inverse dynamics controller, such that the control law is decomposed into two orthogonal subspaces, i.e., the constrained and the unconstrained subspaces. The unconstrained component controls multiple desired tasks with impedance responses. The constrained space controller maintains the contact subject to unknown external disturbances, without the use of any force/torque sensing at the contact points. By explicitly modelling the external force, our controller is robust to external disturbances and errors arising from incorrect dynamic model information. The main contributions of this paper include (1) incorporating an impedance controller to control external disturbances and allow impedance shaping to adjust the behaviour of the motion under external disturbances, (2) optimising contact forces within the constrained subspace that also takes into account the external disturbances without using force/torque sensors at the contact locations. The techniques are evaluated on the ANYmal quadruped platform under a variety of scenarios.
\end{abstract}

\section{INTRODUCTION}

Areas, such as disaster sites and deep mines, are very hazardous for humans to enter due to difficult/unknown terrain. As such potential applications for legged robots can be described allowing those robots to replace the human presence in these areas. The area terrain and characteristics make compliance a necessary feature to protect the robot against unexpected disturbances from either human or environment factors (see Fig. 1). Although position control can produce accurate motion, it relies on perfect knowledge of the environment for planning of the swing leg trajectories, which is impossible to obtain. When the robot is walking on an uneven terrain or slope, the swing leg may strike on an obstacle or land earlier than planned. This impact creates additional perturbations to the whole kinematic chain and might cause the robot to lose balance or stability. Therefore, a flexible torque controlled solution is an ideal solution for unpredictable environments and safe human interaction.

Model-free control of robots for locomotion, such as the virtual model controller, have shown practical advantages by not relying on possibly inaccurate dynamic models, which has been applied on biped [1], quadruped [2] robots, and hydraulically actuated quadrupeds [3]. Model-free controllers typically require tuning of the feedback gains to ensure

Guiyang Xin (gyxin12@csu.edu.cn) is at the School of Informatics, University of Edinburgh, UK and the School of Mechanical and Electrical Engineering, Central South University, China. Hsiu-Chin Lin, Joshua Smith, Oguzhan Cebe, and Michael Mistry are at the School of Informatics, University of Edinburgh, UK.

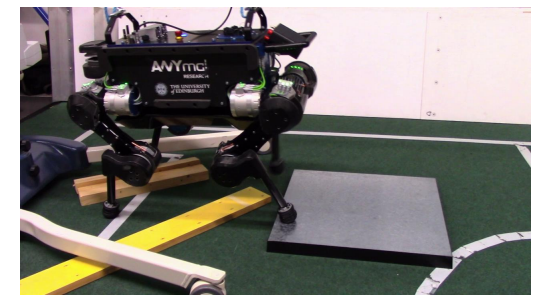

Fig. 1: A quadruped robot, ANYmal, walking over uneven terrain

stability of the system which in an unstable or uncertain environment could produce unstable behaviours.

In contrast, the model-based controller enables legged robots to perform a variety of tasks. Operational space control [4] decomposes the control law into primary and secondary tasks, such that multiple tasks are executed simultaneously. For systems under physical constraints, [5] proposed an approach based on orthogonal decomposition of the control law into constrained and unconstrained subspaces. It was later extended by [6] to enable constraints within the operational space formulation. Being model-based also allows prediction and understanding of the dynamic system at any point in time allowing planners or balancing algorithms to take advantage of this information.

While the model-based controller was originally designed for fixed-based manipulators, it has been extended to floating-based legged robots such as humanoid robots whose control is based on an operational space formulation [7][8] or quadruped locomotion based on QR decomposition [9] or projected inverse dynamics [10].

For the robot to be robust to external disturbance it needs to estimate how much external forces are applied. Previous work estimates the external force based on sensor fusion [11][12]. However, the force/torque sensor data is usually unreliable. In [13], the projected inverse dynamic controller was extended to explicitly measure an estimate of the external disturbances for dual-arm manipulation task, and later was applied to a four arm manipulation task [14].

The optimisation of contact forces is also necessary to achieve the desired task while maintaining the constraints required by the environment for stable motion. For example, when walking on an uneven terrain or during human interactions, the robot needs to regulate the ground reaction force to prevent slipping. This can be done by minimising the torque while ensuring that a desired motion creates feasible interaction forces [9][15] and optimal joint trajectories [16].

We propose a method for hierarchical control of legged robots subject to external disturbance by explicitly modelling the external force. The control law is decomposed into two 
orthogonal subspaces. The unconstrained subspace controls the motion and achieves the desired Cartesian impedance behaviour. The constrained subspace optimises the torque required to maintain the contact subject to unknown, but estimated, external disturbances without the use of force/torque sensing at the contact points. The main contributions include:

- incorporating a Cartesian impedance controller to control disturbances and allow impedance shaping for changing the behaviour under disturbances,

- optimising contact force within the constrained subspace that also takes into account disturbances without using force/torque sensors at the contacts.

By decomposing the control law, we can impose compliance without affecting the constraints, and also optimise the contact force without generating extra motion. While modelbased control of legged systems is potentially more accurate than the position controller and model-free controller, it requires an accurate and precise dynamic model. In this paper, we also show that explicitly modelling the disturbances can relieve this issue. The techniques are evaluated on the ANYmal (Fig. 1) with various scenarios.

\section{BACKGROUND}

Let $\mathbf{q}, \dot{\mathbf{q}}, \ddot{\mathbf{q}} \in \mathbb{R}^{\mathcal{Q}}$ denote the joint positions, velocities, and accelerations of a $(\mathcal{Q}+6)$ degree-of-freedom floating-base system. The dynamics can be expressed in the Lagrangian form

$$
\mathbf{M} \ddot{\mathbf{q}}+\mathbf{h}=\mathbf{B} \tau
$$

where $\boldsymbol{\tau} \in \mathbb{R}^{(\mathcal{Q}+6)}$ is the vector of joint torques, $\mathbf{M} \in$ $\mathbb{R}^{(\mathcal{Q}+6) \times(\mathcal{Q}+6)}$ is the inertia matrix, and $\mathbf{h} \in \mathbb{R}^{(\mathcal{Q}+6)}$ is the vector of centrifugal, and Coriolis effects, and generalised gravitational torque, and

$$
\mathbf{B} \in \mathbb{R}^{(\mathcal{Q}+6) \times(\mathcal{Q}+6)}=\left[\begin{array}{cc}
\mathbf{0}_{6} & \mathbf{0} \\
\mathbf{0} & \mathbf{I}_{\mathcal{Q}}
\end{array}\right]
$$

is the selection matrix. When a robot is in contact with the environment, the end-effector motion may be subject to constraints imposed by the environment. An additional term is added to describe the dynamics under constraints

$$
\mathbf{M} \ddot{\mathbf{q}}+\mathbf{h}=\mathbf{B} \boldsymbol{\tau}+\mathbf{J}_{c}^{\top} \boldsymbol{\lambda}_{c}
$$

where $\mathbf{J}_{c} \in \mathbb{R}^{\mathcal{K} \times(\mathcal{Q}+6)}$ is the constraint Jacobian that describes $\mathcal{K}$ linearly independent constraints, and $\boldsymbol{\lambda}_{c} \in \mathbb{R}^{\mathcal{K}}$ is the constraint forces due to contact that satisfy

$$
\mathbf{J}_{c} \dot{\mathbf{q}}=\mathbf{0} \text { and } \mathbf{J}_{c} \ddot{\mathbf{q}}+\dot{\mathbf{J}}_{c} \dot{\mathbf{q}}=\mathbf{0} \text {. }
$$

\section{A. Projected Inverse Dynamics}

The projected inverse dynamics framework proposed in [5], allows the dynamics equation in (2) to be decomposed into constrained and unconstrained components;

$$
\boldsymbol{\tau}=\mathbf{P} \boldsymbol{\tau}+(\mathbf{I}-\mathbf{P}) \boldsymbol{\tau}
$$

where $\mathbf{P} \in \mathbb{R}^{(\mathcal{Q}+6) \times(\mathcal{Q}+6)}=\mathbf{I}-\mathbf{J}_{c}^{+} \mathbf{J}_{c}$ is the orthogonal projection matrix that projects arbitrary vectors onto the null space of the constraint Jacobian $\mathbf{J}_{c}$, and $\mathbf{J}_{c}^{+}$is the MoorePenrose pseudo-inverse of $\mathbf{J}_{c}$. Note that the two terms in (4) are orthogonal to each other $\mathbf{P} \boldsymbol{\tau} \perp(\mathbf{I}-\mathbf{P}) \boldsymbol{\tau}$ such that the first term $\mathbf{P} \boldsymbol{\tau}$ generates no motion in the constraint space, and the second term $(\mathbf{I}-\mathbf{P}) \boldsymbol{\tau}$ enforces the constraint without generating joint motion.

In [6] a proposed operational space controller for constrained dynamical systems such that the term $\mathbf{P} \boldsymbol{\tau}$ in (4) is replaced by $\mathbf{P} \mathbf{J}_{x}^{\top} \mathbf{F}$, where $\mathbf{J}_{x}$ is the Jacobian matrix that relates the joint space to the task space, and $\mathbf{F}$ is the force applied at the end-effector for the desired acceleration $\ddot{\mathrm{x}}$ :

$$
\mathbf{F}=\boldsymbol{\Lambda}_{c} \ddot{\mathbf{x}}+\boldsymbol{\Lambda}_{c}\left[\mathbf{J}_{x} \mathbf{M}_{c}^{-1}(\mathbf{P h}-\dot{\mathbf{P}} \dot{\mathbf{q}})-\dot{\mathbf{J}}_{x} \dot{\mathbf{q}}\right]=\boldsymbol{\Lambda}_{c} \ddot{\mathbf{x}}+\mathbf{h}_{c}
$$

$\boldsymbol{\Lambda}_{c}=\left(\mathbf{J}_{x} \mathbf{M}_{c}^{-1} \mathbf{P} \mathbf{J}_{x}^{\top}\right)^{-1}$ and $\mathbf{M}_{c}=\mathbf{P M}+\mathbf{I}-\mathbf{P}$ are the constraint consistent operational space and joint space inertia matrix, respectively.

\section{B. Multiple Task Control for Underactuated Systems}

The work in [6] also addresses the problem of hierarchical control in an underactuated system. Given two motion tasks, the control law in a hierarchical order is provided as:

$$
\boldsymbol{\tau}=\mathbf{J}_{1}^{\top} \mathbf{F}_{1}+\mathbf{N}_{1} \mathbf{J}_{2}^{\top} \mathbf{F}_{2}+\mathbf{N}_{u} \boldsymbol{\tau}_{0}
$$

where $\mathbf{J}_{1}, \mathbf{J}_{2}$ are the Jacobin matrix relates the joint space to the primary and the secondary task, $\mathbf{F}_{1}, \mathbf{F}_{2}$ are the desired force, $\mathbf{N}_{1}=\mathbf{I}-\mathbf{J}_{1}^{+} \mathbf{J}_{1}$ is the nullspace projection of $\mathbf{J}_{1}, \mathbf{N}_{u}=\mathbf{I}-\mathbf{J}_{a}^{\top} \mathbf{J}_{a}^{\top \#}$ is the null space projector that is dynamically consistent with all tasks, $\mathbf{J}_{a}=\left[\mathbf{J}_{1}, \mathbf{J}_{2}\right]^{\top}$ is the augmented Jacobian of all tasks, and $\mathbf{J}_{a}^{\top^{\#}}=$ $\left(\mathbf{J}_{a} \mathbf{M}_{c}^{-1} \mathbf{P} \mathbf{J}_{a}^{\top}\right)^{-1} \mathbf{J}_{a} \mathbf{M}_{c}^{-1} \mathbf{P}$ is the dynamically consistent Jacobian pseudo-inverse. Then control law, for the underactuated system, that satisfies the constraint $\tau=\mathbf{B} \tau$ is defined as:

$$
\boldsymbol{\tau}=\left(\mathbf{I}-\mathbf{N}_{u}\left[(\mathbf{I}-\mathbf{B}) \mathbf{N}_{u}\right]^{+}\right)\left(\mathbf{J}_{1}^{\top} \mathbf{F}_{1}+\mathbf{N}_{1} \mathbf{J}_{2}^{\top} \mathbf{F}_{2}\right)
$$

C. Projected Inverse Dynamics with External Disturbances The projected inverse dynamics was later extended to include external disturbances [13], by explicitly add the external force into the dynamic equation, resulting

$$
\mathbf{M} \ddot{\mathbf{q}}+\mathbf{h}=\boldsymbol{\tau}+\mathbf{J}_{c}^{\top} \boldsymbol{\lambda}_{c}+\mathbf{J}_{x}^{\top} \mathbf{F}_{x}
$$

where $\mathbf{J}_{x}$ is the Jacobian that relates the joint-space to the point of disturbances, and $\mathbf{F}_{x}$ is the external force. The motion space component incorporates a Cartesian impedance controller [17][18] to compensate the external disturbances.

$$
\mathbf{P M} \ddot{\mathbf{q}}+\mathbf{P h}=\mathbf{P} \mathbf{J}_{x}^{\top} \mathbf{F}
$$

where $\mathbf{F}=\mathbf{h}_{c}+\boldsymbol{\Lambda}_{c} \ddot{\mathbf{x}}_{d}-\mathbf{D}_{d} \dot{\tilde{\mathbf{x}}}-\mathbf{K}_{d} \tilde{\mathbf{x}}$ is the force needed to achieve the task with the desired Cartesian impedance behaviour. The constrained dynamics is described by

$$
(\mathbf{I}-\mathbf{P})\left(\mathbf{M} \ddot{\mathbf{q}}+\mathbf{h}-\mathbf{J}_{x}^{\top} \mathbf{F}_{x}\right)=\mathbf{J}_{c}^{\top} \mathbf{F}_{c}+\mathbf{J}_{c}^{\top} \boldsymbol{\lambda}_{c}
$$

where $\mathbf{F}_{c}$ is the force needed to maintain the desired constraint force and the contacts. To ensure that the acceleration generated from the constrained space $(\mathbf{I}-\mathbf{P}) \boldsymbol{\tau}$ is consistent with the unconstrained space $\mathbf{P} \boldsymbol{\tau}$, the joint-acceleration in (8) is replaced by $\ddot{\mathbf{q}}=\mathbf{M}_{c}^{-1}(\mathbf{P} \boldsymbol{\tau}-\mathbf{P h}+\dot{\mathbf{P}} \dot{\mathbf{q}})$ resulting in:

$$
\begin{aligned}
& (\mathbf{I}-\mathbf{P})\left[\mathbf{M M}_{c}^{-1}(\mathbf{P} \boldsymbol{\tau}-\mathbf{P h}+\dot{\mathbf{P}} \dot{\mathbf{q}})+\mathbf{h}-\mathbf{J}_{x} \mathbf{F}_{x}\right] \\
& =\mathbf{J}_{c}^{\top} \mathbf{F}_{c}+\mathbf{J}_{c}^{\top} \boldsymbol{\lambda}_{c}
\end{aligned}
$$




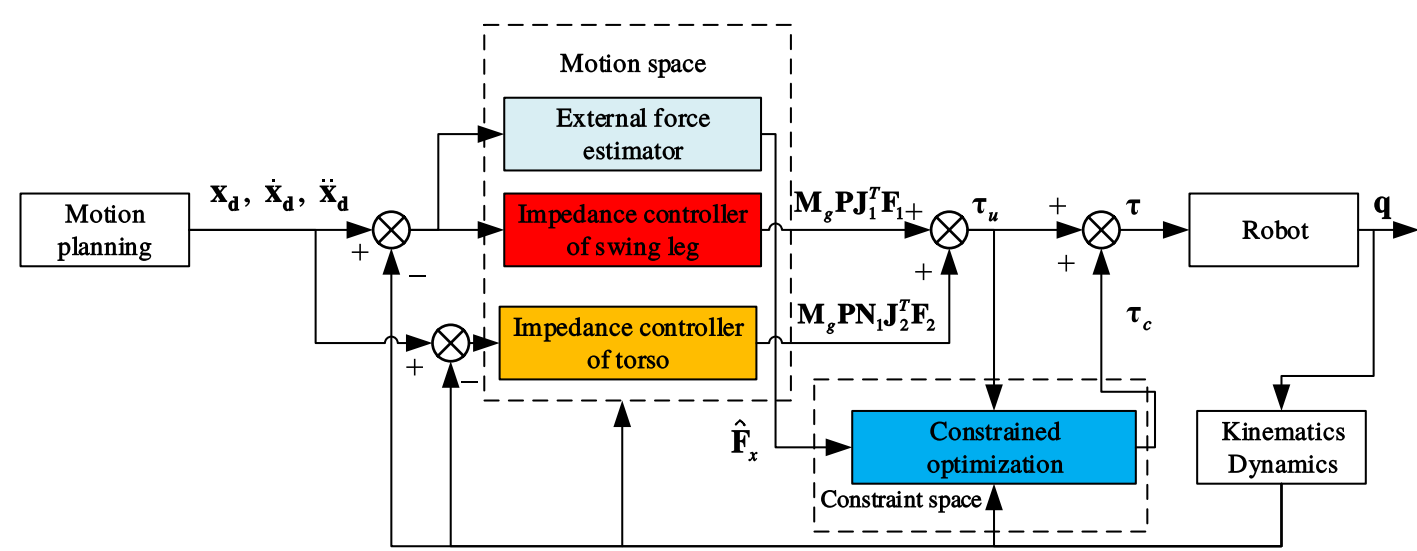

Fig. 2: An overview of the hierarchical controller with optimisation of constrained force for legged systems subject to external disturbances.

In [13], robustness to external disturbances has been shown using fully-actuated, dual-arm manipulation. It was later extended to multi-arm manipulation for underactuated system [14]. In this paper, we extend the formulation in [6][13][14] to enable multiple motion tasks for an underactuated system subject to external disturbances.

\section{METHOD}

A control framework for legged robots to perform multiple motion tasks with respect to constraints in an environment is proposed. We extended the projected inverse dynamics to allow hierarchical motion tasks with constrained space controller for underactuated system in §III-A. The unconstrained subspace controller, which realises the underlying task and impedance behaviour at the swing leg and the torso, is discussed in §III-B while the constrained subspace controller optimises the ground reaction force against external disturbance is discussed in §III-C.

\section{A. Projected Inverse Dynamics for Hierarchical Control of an Underactuated System}

We consider two prioritised tasks for legged systems. For example, during normal walking, the primary task is to move the swing foot to the desired position and the secondary task is tracking the torso trajectory, while maintaining desired ground reaction force. This order of tasks is chosen for accurately placing the foot whilst maintaining some control over the balance. The equation for hierarchical control (see §II-B) with constraint force control becomes:

$$
\boldsymbol{\tau}=\mathbf{P}\left(\mathbf{J}_{1}^{\top} \mathbf{F}_{1}+\mathbf{N}_{1} \mathbf{J}_{2}^{\top} \mathbf{F}_{2}\right)+(\mathbf{I}-\mathbf{P}) \mathbf{J}_{c}^{\top} \mathbf{F}_{c}
$$

Legged robots are traditionally modelled as floating base and intrinsically underactuated. Due to underactuation, we cannot generate the desired end-effector forces $\mathbf{F}_{1}, \mathbf{F}_{2}$ via a Jacobian transpose method, i.e. $\mathbf{J}^{\top} \mathbf{F}$ may not equal to $\mathbf{B J}^{\top} \mathbf{F}$. Instead the admissible torques are limited by

$$
\tau=\mathbf{B} \tau
$$

In order to satisfy the above equation, we add a null space component $\mathbf{N}_{u} \tau_{u}$ resulting in:

$$
\boldsymbol{\tau}=\mathbf{P}\left(\mathbf{J}_{1}^{\top} \mathbf{F}_{1}+\mathbf{N}_{1} \mathbf{J}_{2}^{\top} \mathbf{F}_{2}\right)+(\mathbf{I}-\mathbf{P}) \mathbf{J}_{c}^{\top} \mathbf{F}_{c}+\mathbf{N}_{u} \boldsymbol{\tau}_{u}
$$

where $\mathbf{N}_{u}$ is defined in $\S$ II-B. Solving for $\boldsymbol{\tau}_{u}$ (See Appendix A), the control law is

$$
\boldsymbol{\tau}=\mathbf{M}_{g}\left[\mathbf{P}\left(\mathbf{J}_{1}^{\top} \mathbf{F}_{1}+\mathbf{N}_{1} \mathbf{J}_{2}^{\top} \mathbf{F}_{2}\right)+(\mathbf{I}-\mathbf{P}) \mathbf{J}_{c}^{\top} \mathbf{F}_{c}\right]
$$

where $\mathbf{M}_{g}=\mathbf{I}-\mathbf{N}_{u}\left[(\mathbf{I}-\mathbf{B}) \mathbf{N}_{u}\right]^{+}$is a matrix that projects arbitrary torques onto admissible torques that satisfy $\mathbf{J}^{\top} \mathbf{F}=$ BJ $^{\top}$ F. Substituting (12) into the dynamics equation (7), the dynamic equation can be written as

$$
\begin{aligned}
\mathbf{M} \ddot{\mathbf{q}}+\mathbf{h} & =\mathbf{M}_{g}\left[\mathbf{P}\left(\mathbf{J}_{1}^{\top} \mathbf{F}_{1}+\mathbf{N}_{1} \mathbf{J}_{2}^{\top} \mathbf{F}_{2}\right)+(\mathbf{I}-\mathbf{P}) \mathbf{J}_{c}^{\top} \mathbf{F}_{c}\right] \\
& +\mathbf{J}_{c}^{\top} \boldsymbol{\lambda}_{c}+\mathbf{J}_{x}^{\top} \mathbf{F}_{x}
\end{aligned}
$$

We then define the torque for the unconstrained space as $\boldsymbol{\tau}_{u} \equiv \mathbf{M}_{g} \mathbf{P}\left(\mathbf{J}_{1}^{\top} \mathbf{F}_{1}+\mathbf{N}_{1} \mathbf{J}_{2}^{\top} \mathbf{F}_{2}\right)$ and the constrained space as $\boldsymbol{\tau}_{c} \equiv \mathbf{M}_{g}(\mathbf{I}-\mathbf{P}) \mathbf{J}_{c}^{\top} \mathbf{F}_{c}$ throughout this paper.

We aim to apply kinematic constraints to the stance feet, so the constraint Jacobian $\mathbf{J}_{c}$ in (13) is a concatenation of the Jacobian matrix of the stance legs and that the motion cannot generate velocity on the stance feet. This projection $\mathbf{P}=$ $\mathbf{I}-\mathbf{J}_{c}{ }^{+} \mathbf{J}_{c}$ decomposes the control law such that $\boldsymbol{\tau}_{u}$ resolves the desired primary and secondary motion tasks, while $\boldsymbol{\tau}_{c}$ maintains contact force satisfying environment constraints. An overview of the control flow is sketched in Fig. 2.

\section{B. Unconstrained Space Controller}

By multiplying both sides of (13) by $\mathbf{P}$, the dynamics of unconstrained space is

$$
\mathbf{P M} \ddot{\mathbf{q}}+\mathbf{P h}=\mathbf{M}_{g} \mathbf{P}\left(\mathbf{J}_{1}^{\top} \mathbf{F}_{1}+\mathbf{N}_{1} \mathbf{J}_{2}^{\top} \mathbf{F}_{2}\right)+\mathbf{P} \mathbf{J}_{x} \mathbf{F}_{x}
$$

Since $\mathbf{P} \mathbf{J}_{c}=\mathbf{0}$, the contact force $\boldsymbol{\lambda}_{c}$ vanishes from (13). Our primary task is to move the swing foot to a desired position while being compliant to external disturbance. To achieve this, we define $\mathbf{J}_{1} \in \mathbb{R}^{3 \times(\mathcal{Q}+6)}$ to be the jacobian that relates the joint velocities to the swing foot Cartesian velocity, and $\mathbf{F}_{1}$ is the force needed to accomplish the desired task and impedance response of the swing foot.

$$
\mathbf{F}_{1} \in \mathbb{R}^{3}=\mathbf{h}_{c, s}+\boldsymbol{\Lambda}_{c, s} \ddot{\mathbf{x}}_{d, s}-\mathbf{D}_{s} \dot{\tilde{\mathbf{x}}}_{s}-\mathbf{K}_{s} \tilde{\mathbf{x}}_{s}
$$

where $\tilde{\mathbf{x}}_{s}$ is the error between the current swing foot position and the desired swing foot position, $\mathbf{K}_{s}$ and $\mathbf{D}_{s}$ are the desired Cartesian stiffness and damping matrices for the swing foot. $\boldsymbol{\Lambda}_{c, s}$ is the actual operational space inertia matrix 
of the swing foot. Note we do not attempt inertia shaping, which allows us to avoid contact force/torque sensing [18].

For the secondary task, we control the torso position such that the center of mass is above the support polygon. By using $\mathbf{J}_{2} \in \mathbb{R}^{6 \times(\mathcal{Q}+6)}$ which is the Jacobian that relates the joint velocities to the torso velocities, and $\mathbf{F}_{2}$ is the force for tracking the desired torso configuration with impedance response, we can achieve the desired secondary behaviour.

$$
\mathbf{F}_{2} \in \mathbb{R}^{6}=\mathbf{h}_{c, t}+\boldsymbol{\Lambda}_{c, t} \ddot{\mathbf{x}}_{d, t}-\mathbf{D}_{t} \dot{\tilde{\mathbf{x}}}_{t}-\mathbf{K}_{t} \tilde{\mathbf{x}}_{t}
$$

where $\tilde{\mathbf{x}}_{t}$ is the error between the current and the desired torso configuration, $\mathbf{K}_{t}, \mathbf{D}_{t}$ are the desired Cartesian stiffness and damping matrices, and $\boldsymbol{\Lambda}_{c, t}$ is the actual operational space inertia matrix of the torso.

To determine the desired torso position, we use the method in [15] to generate torso trajectory based on weighted average of legs positions. As the external force location and direction is unknown we cannot guarantee the force is only applied to the primary motion task. As such the secondary motion task requires a compliant behaviour as well, which we control as another impedance controller. The final output from the motion controller is defined as:

$$
\boldsymbol{\tau}_{u}=\mathbf{M}_{g} \mathbf{P}\left(\mathbf{J}_{1}^{\top} \mathbf{F}_{1}+\mathbf{N}_{1} \mathbf{J}_{2}^{\top} \mathbf{F}_{2}\right)
$$

When all legs are in contact, we can see that $\mathbf{J}_{1}=\{\emptyset\}$ and $\mathbf{N}_{1}=\mathbf{I}$, and the unconstrained space controller is reduced to an impedance controller at the torso.

\section{Optimal Ground Reaction Force}

In this section, we outline our approach for the constrained controller. The contact force applied by the legs should be sufficient enough to prevent the separation or sliding of the contact against disturbances arising from inertia, gravitational force, or external disturbances. However, this is not a trivial task since the direction of admissible force is not easy to define due to underactuated control, and excessive force may decrease the stability of the robot. To address this issue we incorporate optimisation strategies to seek the minimal contact force needed. An important note is that this is achieved without using force/torque sensing either at the foot contact points, or at a potential disturbance point (e.g. body push). Previously, [13] proposed the solution for a fully-acutated system with surface contacts. We extend it to an underactuated system with point contacts.

1) Constraints: The foot of each stance leg is treated as a point contact on the ground. The contact force includes $\boldsymbol{\lambda}_{c} \in \mathbb{R}^{3}=\left[\lambda_{x}, \lambda_{y}, \lambda_{z}\right]^{\top}$, and we choose the $\mathrm{z}$-axis as the direction normal to the contact surface. A sketch of the ground reaction forces and the corresponding friction cones is illustrated in Fig. 3. The contact force should satisfy:

- unilateral constraints to avoid loss of contact

$$
\lambda_{z} \geq 0
$$

- friction cone constraints to avoid slipping

$$
\mu \lambda_{z} \geq \sqrt{\lambda_{x}^{2}+\lambda_{y}^{2}}
$$

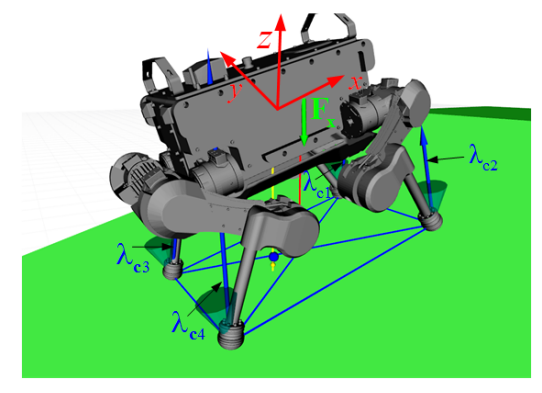

Fig. 3: An illustration of the ground reaction forces $\boldsymbol{\lambda}_{c}$ and their corresponding friction cones (green).

2) Objective Function: Our goal is to find the minimum actuator torque

$$
\underset{\tau}{\operatorname{minimise}} \boldsymbol{\tau}^{\top} \boldsymbol{\tau}
$$

or equivalently,

$$
\underset{\boldsymbol{\tau}}{\operatorname{minimise}} \boldsymbol{\tau}_{u}^{\top} \boldsymbol{\tau}_{u}+2 \boldsymbol{\tau}_{u}^{\top} \boldsymbol{\tau}_{c}+\boldsymbol{\tau}_{c}^{\top} \boldsymbol{\tau}_{c}
$$

By setting $\boldsymbol{\tau}_{c} \equiv \mathbf{M}_{g} \mathbf{J}_{c}^{\top} \mathbf{F}_{c}$, we can guarantee that $\boldsymbol{\tau}_{c}$ lies within the constrained space and $\boldsymbol{\tau}_{c} \perp \boldsymbol{\tau}_{u}$. Since $\boldsymbol{\tau}_{u}^{\top} \boldsymbol{\tau}_{u}$ is a constant during optimisation and $2 \boldsymbol{\tau}_{u}^{\top} \boldsymbol{\tau}_{c}=0$, we can simply minimise $\boldsymbol{\tau}_{c}^{\top} \boldsymbol{\tau}_{c}$. Then the objective function can be reformulated in terms of the unknown variable $\mathbf{F}_{c}$ :

$$
\underset{\mathbf{F}_{c}}{\operatorname{minimise}} \mathbf{F}_{c}^{\top} \mathbf{J}_{c} \mathbf{M}_{g}^{\top} \mathbf{M}_{g} \mathbf{J}_{c}^{\top} \mathbf{F}_{c}
$$

3) Constrained Optimisation problem: For the constrained subspace, we derive the constrained subspace dynamics following similar procedure in [13], but for the underactuated system, i.e. multiply both sides of (13) by $(\mathbf{I}-\mathbf{P})$ and move the external force to the left side,

$$
(\mathbf{I}-\mathbf{P})\left(\mathbf{M} \ddot{\mathbf{q}}+\mathbf{h}-\mathbf{J}_{x} \mathbf{F}_{x}\right)=\mathbf{M}_{g} \mathbf{J}_{c}^{\top} \mathbf{F}_{c}+\mathbf{J}_{c}^{\top} \boldsymbol{\lambda}_{c}
$$

To ensure that the acceleration generated from the constrained space controller $\tau_{c}$ is consistent with the unconstrained space controller $\tau_{u}$, the joint-acceleration in (19) is replaced by $\ddot{\mathbf{q}}=\mathbf{M}_{c}^{-1}\left(\boldsymbol{\tau}_{u}-\mathbf{P h}+\dot{\mathbf{P}} \dot{\mathbf{q}}\right)$ in [6] resulting in:

$$
\begin{aligned}
& (\mathbf{I}-\mathbf{P})\left[\mathbf{M M}_{c}^{-1}\left(\boldsymbol{\tau}_{u}-\mathbf{P h}+\dot{\mathbf{P}} \dot{\mathbf{q}}\right)+\mathbf{h}-\mathbf{J}_{x} \mathbf{F}_{x}\right] \\
& =\mathbf{M}_{g} \mathbf{J}_{c}^{\top} \mathbf{F}_{c}+\mathbf{J}_{c}^{\top} \boldsymbol{\lambda}_{c}
\end{aligned}
$$

The external force $\mathbf{F}_{x}$ is replaced by estimated external force

$$
\hat{\mathbf{F}}_{x}=\hat{\boldsymbol{\Lambda}}_{c} \ddot{\tilde{\mathbf{x}}}+\mathbf{D}_{d} \dot{\tilde{\mathbf{x}}}+\mathbf{K}_{d} \tilde{\mathbf{x}}
$$

A key insight, is that the external force $\hat{\mathbf{F}}_{x}$ is estimated based on position error, and thus we do not require force/torque sensing at the contacts. The optimisation problem is to find the optimal contact force $\boldsymbol{\lambda}_{c}$ that minimises the objective function (18) while satisfying the unilateral constraints (16) and the friction cone constraints (17) at the contact points, and balance out the external forces.

$$
\begin{aligned}
\underset{\mathbf{F}_{c}}{\operatorname{minimise}} & \mathbf{F}_{c}^{\top} \mathbf{J}_{c} \mathbf{M}_{g}^{\top} \mathbf{M}_{g} \mathbf{J}_{c}^{\top} \mathbf{F}_{c} \\
\text { subject to } & \lambda_{z}^{i} \geq 0 \\
& \mu \lambda_{z}^{i} \geq \sqrt{\left(\lambda_{x}^{i}\right)^{2}+\left(\lambda_{y}^{i}\right)^{2}}
\end{aligned}
$$


where the superscript $i$ denotes the contact of the $i^{t h}$ leg. We multiply both sides of (20) by $\left(\mathbf{J}_{c}^{\top}\right)^{+}$, and move the term for external disturbance to the left hand side, resulting

$$
\begin{array}{r}
\left(\mathbf{J}_{c}^{\top}\right)^{+}(\mathbf{I}-\mathbf{P})\left[\mathbf{M M}_{c}^{-1}\left(\boldsymbol{\tau}_{u}-\mathbf{P h}+\dot{\mathbf{P}} \dot{\mathbf{q}}\right)+\mathbf{h}-\mathbf{J}_{x}^{\top} \hat{\mathbf{F}}_{x}\right] \\
=\left(\mathbf{J}_{c}^{\top}\right)^{+} \mathbf{M}_{g} \mathbf{J}_{c}^{\top} \mathbf{F}_{c}+\boldsymbol{\lambda}_{c}
\end{array}
$$

We define

$$
\begin{aligned}
\boldsymbol{\eta} & =\left(\mathbf{J}_{c}^{\top}\right)^{+}(\mathbf{I}-\mathbf{P})\left[\mathbf{M M}_{c}^{-1}\left(\boldsymbol{\tau}_{u}-\mathbf{P h}+\dot{\mathbf{P}} \dot{\mathbf{q}}\right)+\mathbf{h}-\mathbf{J}_{x}^{\top} \hat{\mathbf{F}}_{x}\right] \\
\boldsymbol{\rho} & =\left(\mathbf{J}_{c}^{\top}\right)^{+} \mathbf{M}_{g} \mathbf{J}_{c}^{\top} \mathbf{F}_{c}
\end{aligned}
$$

where $\boldsymbol{\eta}$ can be interpreted as the sum of all external force in the constrained space, and $\rho$ is the admissible commanded force. The relationship between the contact force, the admissible commanded force, and the external force can be described by $\boldsymbol{\eta}=\boldsymbol{\rho}+\boldsymbol{\lambda}_{c}$. We can then reformulate the constraint optimisation problem without the contact force.

$$
\begin{aligned}
& \underset{\mathbf{F}_{c}}{\operatorname{minimise}} \mathbf{F}_{c}^{\top} \mathbf{J}_{c} \mathbf{M}_{g}^{\top} \mathbf{M}_{g} \mathbf{J}_{c}^{\top} \mathbf{F}_{c} \\
& \text { subject to } \eta_{z}^{i}-\rho_{z}^{i} \geq 0 \\
& \mu\left(\eta_{z}^{i}-\rho_{z}^{i}\right) \geq \sqrt{\left(\eta_{x}^{i}-\rho_{x}^{i}\right)^{2}+\left(\eta_{y}^{i}-\rho_{y}^{i}\right)^{2}}
\end{aligned}
$$

Remark By setting $\boldsymbol{\tau}_{c} \equiv \mathbf{M}_{g} \mathbf{J}_{c} \mathbf{F}_{c}$, the resulting torque satisfies $\tau=\mathbf{B} \tau$ and $\tau_{u} \perp \boldsymbol{\tau}_{c}$, so we can relax the equality constraint proposed in [19].

Standard constrained optimisation such as the active-set method iteratively solves a sequence of equality and inequality constraints. We simplify the constrained problem by changing the cost function so that some of the constraints are inherently satisfied by the function formulation that reduces the number of active constraints. Simplicity and efficiency is always favourable for real-time control of robots.

Finally, the friction cone constraints in (17) are quadratic inequality constraints and not realistic to solve for real-time control. We approximate the constraints with linearised friction cones of 4 edges. The constraint optimisation problem was then solved using quadratic programming [20].

Quadratic programming aims to minimise $\mathbf{x}^{\top} \mathbf{Q} \mathbf{x}+\mathbf{q}^{\top} \mathbf{x}$ subject to some equality and inequality constraints where $\mathbf{x}$ is the decision variables and $\mathbf{Q}$ is the quadratic cost function. Standard solver requires that $\mathbf{Q}$ to be positive definite ( $\mathbf{Q}=\mathbf{J}_{c} \mathbf{M}_{g}^{\top} \mathbf{M}_{g} \mathbf{J}_{c}^{\top}$ in our case). Since $\mathbf{M}_{g}$ projects out torques that are not admissible, our $\mathbf{Q}$ is rank deficient, and cannot be solved by standard QP solver. Although it is solvable by sequential quadratic programming, it is more computationally expensive. Our controller is running at roughly $2.5 \mathrm{~ms}$; as a trade-off between accuracy and speed, we add a regularisation term to the cost function $\mathbf{Q}=\mathbf{J}_{c} \mathbf{M}_{g}^{\top} \mathbf{M}_{g} \mathbf{J}_{c}^{\top}+\epsilon \mathbf{I}$. $\left(\epsilon=10^{-10}\right.$ in the experiment $)$ We then reduce the friction coefficients, to be smaller than what they should be, in order to ensure that the constraints are still satisfied even with potential numerical errors due to the additional regularisation term.

In summary, we proposed a model-based, hierarchical controller for legged system subject to external disturbances. The motion controller achieves the desired swing leg and torso trajectories, while the constrained controller maintains the desired contact force. By explicitly estimating the external force, our controller is robust to disturbances.

Remark This model-based control is traditionally dependent on the accuracy of the dynamic model, however, our estimated external force in (21) includes not only the human interactions, but also encapsulates the model-error. Therefore, the resulting constraint force is robust to model-error.

Proof: Let $\boldsymbol{\Lambda}_{c}$ and $\hat{\boldsymbol{\Lambda}}_{c}$ be the actual and the estimated operational space inertia matrix, $\mathbf{h}_{c}$ and $\hat{\mathbf{h}}_{c}$ be the actual and the estimated operational space centrifugal, Coriolis and gravity, the operational space dynamics is

$$
\boldsymbol{\Lambda}_{c} \ddot{\mathbf{x}}+\mathbf{h}_{c}=\mathbf{F}+\mathbf{F}_{x}
$$

where $\mathbf{F}$ is the force needed for the desired task and $\mathbf{F}_{x}$ is the actual external force. Without inertia shaping [18], the force that realises the task and impedance response is

$$
\mathbf{F}=\hat{\mathbf{h}}_{c}+\hat{\mathbf{\Lambda}}_{c} \ddot{\mathbf{x}}_{d}-\mathbf{D}_{d} \dot{\tilde{\mathbf{x}}}-\mathbf{K}_{d} \tilde{\mathbf{x}}
$$

Substitute (27) into (26), resulting

$$
\boldsymbol{\Lambda}_{c} \ddot{\mathbf{x}}+\mathbf{h}_{c}=\hat{\mathbf{h}}_{c}+\hat{\boldsymbol{\Lambda}}_{c} \ddot{\mathbf{x}}_{d}-\mathbf{D}_{d} \dot{\tilde{\mathbf{x}}}-\mathbf{K}_{d} \tilde{\mathbf{x}}+\mathbf{F}_{x}
$$

Adding $\hat{\boldsymbol{\Lambda}}_{c} \ddot{\mathbf{x}}-\hat{\boldsymbol{\Lambda}}_{c} \ddot{\mathbf{x}}$, to the left side

$\boldsymbol{\Lambda}_{c} \ddot{\mathbf{x}}+\hat{\boldsymbol{\Lambda}_{c}} \ddot{\mathbf{x}}-\hat{\boldsymbol{\Lambda}_{c}} \ddot{\mathbf{x}}+\mathbf{h}_{c}=\hat{\mathbf{h}}_{c}+\hat{\boldsymbol{\Lambda}}_{c} \ddot{\mathbf{x}}_{d}-\mathbf{D}_{d} \dot{\tilde{\mathbf{x}}}-\mathbf{K}_{d} \tilde{\mathbf{x}}+\mathbf{F}_{x}$

Simply the above equations

$$
\hat{\boldsymbol{\Lambda}}_{c} \ddot{\tilde{\mathbf{x}}}+\mathbf{D}_{d} \dot{\tilde{\mathbf{x}}}+\mathbf{K}_{d} \tilde{\mathbf{x}}=\left(\hat{\mathbf{h}}_{c}-\mathbf{h}_{c}\right)+\left(\hat{\boldsymbol{\Lambda}}_{c}-\boldsymbol{\Lambda}_{c}\right) \ddot{\mathbf{x}}+\mathbf{F}_{x}
$$

Note that, the left hand side of the equation is equal to our estimated external force in (21). We can see that, this term arises from the true external force $\mathbf{F}_{x}$ and the modelling error $\hat{\mathbf{h}}_{c}-\mathbf{h}_{c}$ and $\hat{\boldsymbol{\Lambda}}_{c}-\boldsymbol{\Lambda}_{c}$.

Remark In fact, when there is zero external disturbance, the left side of (28) encapsulates only information about modelling error, which can be used to update the model. We plan to incorporate this in a future work.

\section{Evaluation}

We conduct experiments using ANYmal, a torque-controlled quadruped robot made by ANYbotics ${ }^{1}$. The robot weights approximately $35 \mathrm{~kg}$ and has 12 joints actuated by Series Elastic Actuators (SEAs). Currently, the soft realtime control cycle is $2.5 \mathrm{~ms}$. The control software is developed based on Robot Operating System (ROS). A video of the experimental results can be found in https://mistrygroup.bitbucket.io/pages/videos.html

\section{A. Optimising contact force with estimated external force}

We would like to highlight the benefit of optimising ground reaction force with estimated external force. Note that, without incorporating the external force term in the optimisation, it could be dangerous for human to interact with the robot. Therefore this experiment has been done in simulation.

In Fig. 4, external forces are applied to the torso (green arrow). The desired and the actual the centre-of-mass projected

\footnotetext{
${ }^{1}$ See http://www.anybotics.com/
} 

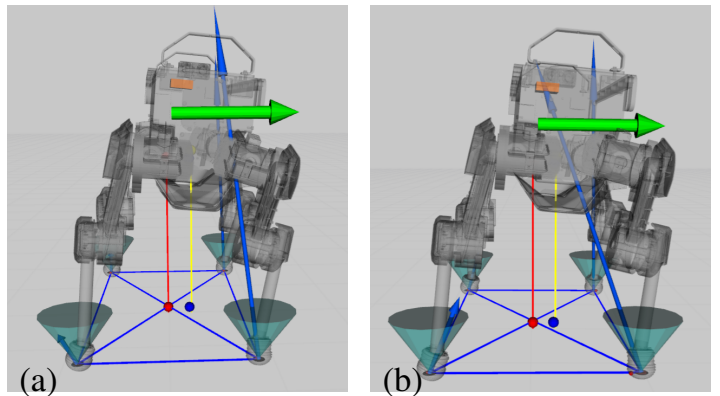

Fig. 4: Examples of constrained optimisation including/excluding the estimated of external force. The green arrows are the external force, the red and blue solid circles are the center-of-mass projected on the ground, the blue arrows are the contact force. The figures show (a) without the external force term and (b) with the external force term.

on the ground are shown as red and blue solid circle on the ground. The blue arrows are the actual ground reaction force.

In Fig. 4(a), the estimated external force term $\hat{\mathbf{F}}_{x}$ is not included in the optimisation in (25). We can see that the ground reaction force of the left foot is at the edge of the friction cone. Continuously applying external force will cause the robot to slip. In contrast, if the external force term is included, as described in (25), we can see that the ground reaction force is maintain within the friction cone from Fig. 4(b). (see supplementary video)

\section{B. Withstanding model-error}

In this experiment, we set up an extreme case in simulation to verify that our proposed method is robust to model-error. At the beginning of the experiment, the robot is standing on a slope. We choose very high PD gains and slowly decrease the gravity compensation term from $100 \%$ to $0 \%$.

The result of the synthetic model-error is shown in Fig. 5, where the $\mathrm{x}$-axis is time (s) and the $\mathrm{y}$-axis is force norm (N). The colours denote magnitudes of the estimated external force (blue), force needed for the motion controller (red), and the gravity compensation term (yellow). We can observe that the gravity compensation term decreases while the external force increases. This is expected since without interaction and acceleration, the estimated external force should reduce to $\hat{\mathbf{F}}_{x}=\hat{\mathbf{h}}-\mathbf{h}$ from (28) and (21).

The controller has no gravity compensation at the end of the simulation, but the ground reaction force is still inside of the friction cone. This is reasonable since $\hat{\mathbf{F}}_{x}$ is included in the optimisation. We can also see that the force for the motion controller (red) is approximately the same throughout the simulation. This is also expected since the $\mathbf{F}$ increases due to position and velocity errors.

\section{Shaping Stiffness Ellipsoid}

In this experiment, we demonstrate the advantage of shaping the stiffness ellipsoid. A well known and widely used condition for static balancing is that the centre-of-mass projected on the ground should be inside of the support polygon. In a real world scenario, external disturbances may be coming from arbitrary directions and could cause the centre-of-mass to fall outside of the stability margin. Due to the mechanical design of the robot, the support polygon might be much

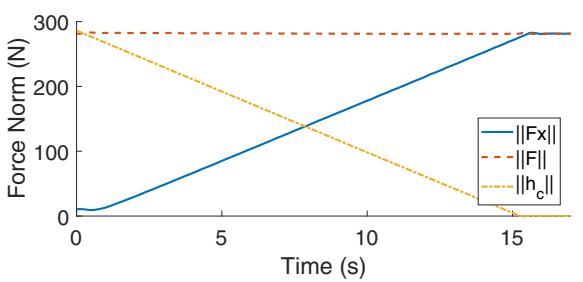

Fig. 5: Experiment on synthetic model-error. The x-axis is time and the $y$-axis is force norm $(\mathrm{N})$. The colours denote magnitudes of the estimated external force (blue), force needed for the motion controller (red), and the gravity compensation term (yellow).
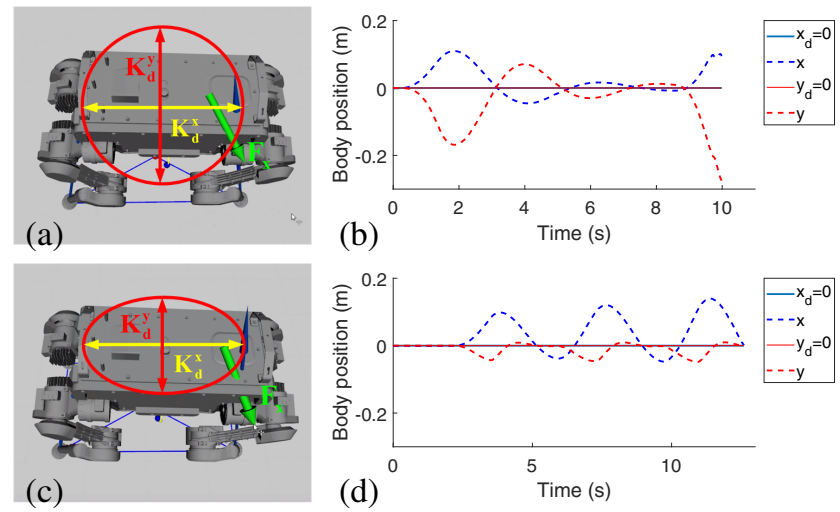

Fig. 6: Experiment of shaping stiffness ellipsoid. (a) and (c) show the stiffness ellipsoid (red circle) of the torso. (a) has equal stiffness in all directions while (c) has higher stiffness along the coronal plane, (b)(d) are the resulting torso positions in sagittal (blue) and coronal (red) direction, the solid and dash lines are the desired and the actual positions.

narrower in some directions; e.g., the coronal plane (red) is much narrower than the sagittal (blue) plane for ANYmal in Fig. 6(b), and the disturbances alone in this direction can cause the robot to lose balance and fall over. This problem can be alleviated by shaping the stiffness ellipsoid based on the balance stability margin.

To demonstrate this, we first set equal stiffness in all linear directions for the torso, the stiffness ellipsoid is shown in Fig. 6(a). An external force (green arrow) is applied at the corner of the torso and Fig. 6(b) shows the torso position in sagittal (blue) and coronal (red) direction. We can see that the positions of the torso in these two directions are moving with approximately the same magnitude.

Then, Fig. 6(c) shows the stiffness ellipsoid after increasing the stiffness for coronal direction, so the resulting stiffness ellipsoid matches the shape of the balance stability margin. Then we apply external disturbances at the same corner of the torso. In Fig. 6(d), the motion along the coronal plane (red) is less affected by the external force and hence more stable in balance. The experiment is performed in both simulation and real robot. (Please see supplementary video)

\section{Hierarchical Impedance Control}

In this experiment, we would like to show our hierarchical impedance controller under external disturbances. The task of the robot is to maintain the swing foot at a desired position in the air, while the torso is tracking the desired center-of- 

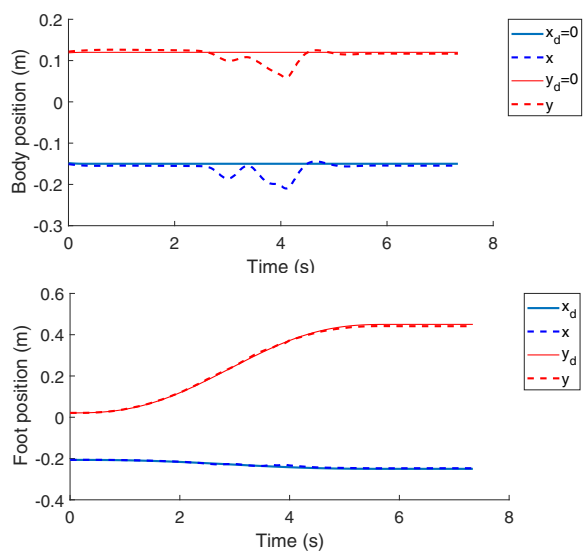

Fig. 7: Experiment of disturbing the torso while the swing leg is moving to its desired position. The figures are (a) position of the body and (b) position of the swing leg
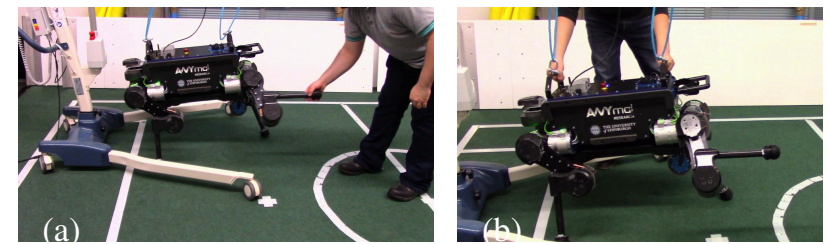

Fig. 8: Experiment of disturbing the foot of ANYmal. (a) force on the swing leg. (b) force on the torso.

mass trajectory. In the real world scenario disturbances are coming from arbitrary directions, which might hit the leg or the body, as a result we would like to see how the robot reacts with these random disturbances.

At the beginning of the simulation, the robot is standing on a flat terrain, and then the swing leg slowly moves to a desired height while external force is supplied at the torso. The torso position is shown in Fig. 7(a) and the swing foot position is shown in Fig. 7(b). The solid lines are the desired positions and the dash lines are the actual positions, and the colours denote $\mathrm{x}$ (blue) and $\mathrm{y}$ (red) positions. The interaction happens around $t=2.5$, and we can see that, the torso position is disturbed while the swing foot position is still tracking the desired height.

We also perform a similar experiment on the real robot, and a human subject is able to randomly interact with the swing leg and the torso, as shown in Fig. 8. Readers can see the complete process of this experiment from the corresponding video of this paper.

\section{E. Walking on Rough Terrain}

Compliance is essential for the robot to walk over uneven terrain, since the swing leg may strike an obstacle and land much earlier than planned. Without compliance, this creates additional disturbances to the robot and might cause the robot to lose its balance. In this experiment, we demonstrate the usefulness of the impedance controller at the swing foot by walking through an area with random blocks (Fig. 9).

For motion planning, we first calculate the desired swing foot position using the method in [21]. A trajectory of desired foot position is a linear interpolation between the initial foot position, desired foot height, and the desired foot position.

Our controller realises the swing foot has struck on the
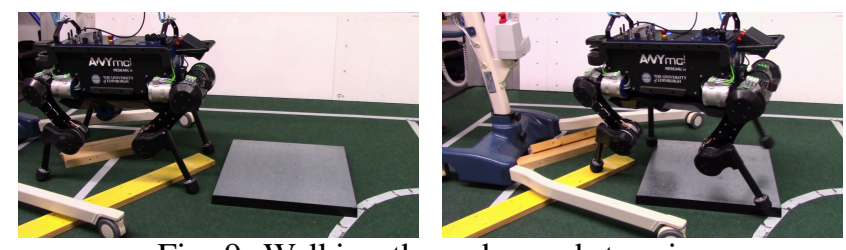

Fig. 9: Walking through rough terrain.
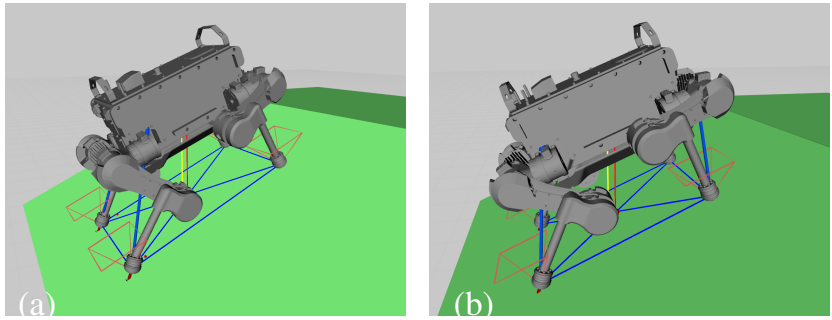

Fig. 10: Standing on a 15 degree slope (a) without constrained optimisation (b) with constrained optimisation
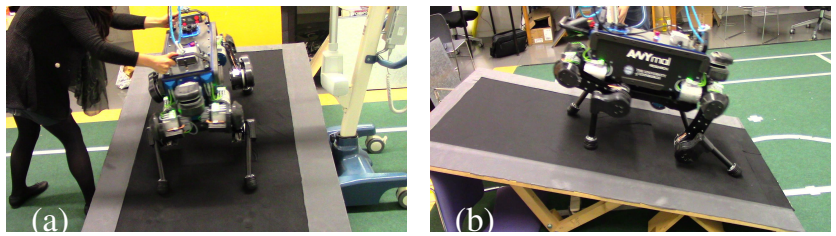

Fig. 11: On a 15 degree slope. (a) interaction (b) walking

obstacle if the swing foot velocities has a large error between the expected and actual swing foot velocities. If this occurs then our controller will consider it is a stance leg in the next time step. Therefore we don't need to rely on external force/torque sensors to detect the collision.

\section{F. Standing and Walking on Slope}

Previous work of using projected inverse dynamics for quadruped locomotion in [10] ignores the constrained space torque, i.e. $\tau_{c}$. This approach works well on flat terrain since the weight of the robot is sufficient to keep the ground reaction force inside of the friction cone. To demonstrate the importance of optimising the constrained force, we examine the scenario on a 15 degree slope.

In Fig. 10 (a), without adding the constrained space controller, the ground reaction forces (blue) fall outside of the friction cones. In the supplementary video, we can see that the feet continuously slides on the slope and eventually fell. In Fig. 10 (b) with our optimisation the ground reaction force is well maintained. The robot is able to walk up the slope and is robust to disturbances.

\section{CONClusion}

In this paper, a method for hierarchical control of legged system subject to external disturbance is proposed. The control framework is based on extending the projected inverse dynamics controller to hierarchical motion control with constrained space control for underactuation. The unconstrained component controls the motion of the swing leg and the torso while achieving the desired Cartesian impedance behaviour. The constrained space controller optimises the torque required to maintain the contact subject to unknown external disturbances, and this is done without use of any force/torque sensing at the feet or disturbance location. Although our 
experimental platform is a quadruped robot, the proposed theory is generic for legged systems with a hierarchy of tasks.

Our experimental results have demonstrated the robustness of the proposed method in various scenarios showing that we can achieve the desired motion while optimising the contact force against disturbances. We have also proven that the estimated external force encapsulates the force arisen from human interaction and model-error.

In this paper, we focus on the control framework and optimisation of the contact forces. In the future, we will incorporate a more robust motion planning (e.g., foot step, swing trajectory) against disturbances.

\section{ACKNOWLEDGEMENT}

This work was funded by the European Commission Horizon 2020 Work Programme: CogIMon ICT-23-2014 644727 and THING ICT-2017-1 780883, as well as EPSRCs RAI Hubs for Extreme and Challenging Environments: ORCA EPR026173/1 and NCNR EPR02572X/1, and the China Scholarship Council (Grant No. 201606370091).

\section{APPENDIX}

\section{A. Deriving the hierarchical control formulation}

From (11), we add an additional term $\mathbf{N}_{u} \boldsymbol{\tau}_{u}$ resulting

$$
\boldsymbol{\tau}=\mathbf{P}\left(\mathbf{J}_{1}^{\top} \mathbf{F}_{1}+\mathbf{N}_{1} \mathbf{J}_{2}^{\top} \mathbf{F}_{2}\right)+(\mathbf{I}-\mathbf{P}) \mathbf{J}_{c}^{\top} \mathbf{F}_{c}+\mathbf{N}_{u} \boldsymbol{\tau}_{u}
$$

Our goal is to have $\tau=\mathbf{B} \tau$.

$$
\begin{aligned}
& \mathbf{P}\left(\mathbf{J}_{1}^{\top} \mathbf{F}_{1}+\mathbf{N}_{1} \mathbf{J}_{2}^{\top} \mathbf{F}_{2}\right)+(\mathbf{I}-\mathbf{P}) \mathbf{J}_{c}^{\top} \mathbf{F}_{c}+\mathbf{N}_{u} \boldsymbol{\tau}_{u} \\
& =\mathbf{B P}\left(\mathbf{J}_{1}^{\top} \mathbf{F}_{1}+\mathbf{N}_{1} \mathbf{J}_{2}^{\top} \mathbf{F}_{2}\right)+\mathbf{B}(\mathbf{I}-\mathbf{P}) \mathbf{J}_{c}^{\top} \mathbf{F}_{c}+\mathbf{B} \mathbf{N}_{u} \boldsymbol{\tau}_{u}
\end{aligned}
$$

Move $\mathbf{N}_{u} \boldsymbol{\tau}_{u}$ to the right side,

$$
\begin{gathered}
(\mathbf{I}-\mathbf{B}) \mathbf{P}\left(\mathbf{J}_{1}^{\top} \mathbf{F}_{1}+\mathbf{N}_{1} \mathbf{J}_{2}^{\top} \mathbf{F}_{2}\right)+(\mathbf{I}-\mathbf{B})(\mathbf{I}-\mathbf{P}) \mathbf{J}_{c}^{\top} \mathbf{F}_{c} \\
=-(\mathbf{I}-\mathbf{B}) \mathbf{N}_{u} \boldsymbol{\tau}_{u}
\end{gathered}
$$

Solving for $\tau_{u}$

$$
\begin{aligned}
& \boldsymbol{\tau}_{u}=-\left[(\mathbf{I}-\mathbf{B}) \mathbf{N}_{u}\right]^{+} \\
& \quad\left[(\mathbf{I}-\mathbf{B}) \mathbf{P}\left(\mathbf{J}_{1}^{\top} \mathbf{F}_{1}+\mathbf{N}_{1} \mathbf{J}_{2}^{\top} \mathbf{F}_{2}\right)+(\mathbf{I}-\mathbf{B})(\mathbf{I}-\mathbf{P}) \mathbf{J}_{c}^{\top} \mathbf{F}_{c}\right]
\end{aligned}
$$

Substitute $\tau_{u}$ back into the original equation

$$
\begin{aligned}
\boldsymbol{\tau} & =\mathbf{P}\left(\mathbf{J}_{1}^{\top} \mathbf{F}_{1}+\mathbf{N}_{1} \mathbf{J}_{2}^{\top} \mathbf{F}_{2}\right)+(\mathbf{I}-\mathbf{P}) \mathbf{J}_{c}^{\top} \mathbf{F}_{c} \\
& -\mathbf{N}_{u}\left[(\mathbf{I}-\mathbf{B}) \mathbf{N}_{u}\right]^{+}(\mathbf{I}-\mathbf{B}) \mathbf{P}\left(\mathbf{J}_{1}^{\top} \mathbf{F}_{1}+\mathbf{N}_{1} \mathbf{J}_{2}^{\top} \mathbf{F}_{2}\right) \\
& -\mathbf{N}_{u}\left[(\mathbf{I}-\mathbf{B}) \mathbf{N}_{u}\right]^{+}(\mathbf{I}-\mathbf{B})(\mathbf{I}-\mathbf{P}) \mathbf{J}_{c}^{\top} \mathbf{F}_{c} \\
& =\left(\mathbf{I}-\mathbf{N}_{u}\left[(\mathbf{I}-\mathbf{B}) \mathbf{N}_{u}\right]^{+}(\mathbf{I}-\mathbf{B})\right) \mathbf{P}\left(\mathbf{J}_{1}^{\top} \mathbf{F}_{1}+\mathbf{N}_{1} \mathbf{J}_{2}^{\top} \mathbf{F}_{2}\right) \\
& +\left(\mathbf{I}-\mathbf{N}_{u}\left[(\mathbf{I}-\mathbf{B}) \mathbf{N}_{u}\right]^{+}(\mathbf{I}-\mathbf{B})\right)(\mathbf{I}-\mathbf{P}) \mathbf{J}_{c}^{\top} \mathbf{F}_{c}
\end{aligned}
$$

Since $(\mathbf{I}-\mathbf{B})$ is a projection, $\mathbf{I}-\mathbf{N}_{u}\left[(\mathbf{I}-\mathbf{B}) \mathbf{N}_{u}\right]^{+}(\mathbf{I}-\mathbf{B})=$ $\mathbf{I}-\mathbf{N}_{u}\left[(\mathbf{I}-\mathbf{B}) \mathbf{N}_{u}\right]^{+}$. Let $\mathbf{M}_{g}=\mathbf{I}-\mathbf{N}_{u}\left[(\mathbf{I}-\mathbf{B}) \mathbf{N}_{u}\right]^{+}$, the control law is

$$
\boldsymbol{\tau}=\mathbf{M}_{g}\left[\mathbf{P}\left(\mathbf{J}_{1}^{\top} \mathbf{F}_{1}+\mathbf{N}_{1} \mathbf{J}_{2}^{\top} \mathbf{F}_{2}\right)+(\mathbf{I}-\mathbf{P}) \mathbf{J}_{c}^{\top} \mathbf{F}_{c}\right]
$$

\section{REFERENCES}

[1] J. Pratt, C.-M. Chew, A. Torres, P. Dilworth, and G. Pratt, "Virtual model control: An intuitive approach for bipedal locomotion," The International Journal of Robotics Research, vol. 20, no. 2, pp. 129143, 2001.

[2] M. Hutter, M. Gehring, M. Bloesch, C. D. Remy, R. Y. Siegwart, and M. A. Hoepflinger, "Starleth: A compliant quadrupedal robot for fast, efficient, and versatile locomotion," 2012.

[3] T. Boaventura, C. Semini, J. Buchli, M. Frigerio, M. Focchi, and D. G. Caldwell, "Dynamic torque control of a hydraulic quadruped robot," in IEEE International Conference on Robotics and Automation, 2012, pp. 1889-1894.

[4] O. Khatib, "A unified approach for motion and force control of robot manipulators: The operational space formulation," IEEE J. Robotics \& Automation, vol. 3, no. 1, pp. 43-53, 1987.

[5] F. Aghili, "A unified approach for inverse and direct dynamics of constrained multibody systems based on linear projection operator: applications to control and simulation," IEEE Transactions on Robotics, vol. 21 , no. 5 , pp. 834-849, 2005.

[6] M. Mistry and L. Righetti, "Operational space control of constrained and underactuated systems," Robotics: Science and systems, 2011.

[7] L. Sentis, J. Park, and O. Khatib, "Compliant control of multicontact and center-of-mass behaviors in humanoid robots," IEEE Transactions on Robotics, vol. 26, no. 3, pp. 483-501, 2010.

[8] A. Herzog, L. Righetti, F. Grimminger, P. Pastor, and S. Schaal, "Balancing experiments on a torque-controlled humanoid with hierarchical inverse dynamics," in IEEE International Conference on Intelligent Robotics Systems, 2014.

[9] L. Righetti, J. Buchli, M. Mistry, M. Kalakrishnan, and S. Schaal, "Optimal distribution of contact forces with inverse-dynamics control," The International Journal of Robotics Research, vol. 32, no. 3, pp. 280-298, 2013.

[10] A. W. Winkler, F. Farshidian, D. Pardo, M. Neunert, and J. Buchli, "Fast trajectory optimization for legged robots using vertex-based zmp constraints," IEEE Robotics and Automation Letters, vol. 2, pp. 22012208, july 2017.

[11] F. Iida and R. Pfeifer, "Sensing through body dynamics," Robotics and Autonomous Systems, vol. 54, no. 8, pp. 631-640, 2006.

[12] S. Faraji and A. J. Ijspeert, "Designing a virtual whole body tactile sensor suit for a simulated humanoid robot using inverse dynamics," in IEEE International Conference on Intelligent Robots and Systems, 2016, pp. 5564-5571.

[13] H.-C. Lin, J. Smith, K. Kouhkiloui Babarahmati, N. Dehio, and M. Mistry, "A projected inverse dynamics approach for multi-arm cartesian impedance control," in IEEE International Conference on Robotics and Automation, 2018.

[14] N. Dehio, J. Smith, D. L. Wigand, G. Xin, H.-C. Lin, J. J. Steil, and M. Mistry, "Modeling and control of multi-arm and multi-leg robots: Compensating for object dynamics during grasping," in IEEE International Conference on Robotics and Automation, 2018.

[15] C. Gehring, S. Coros, M. Hutter, M. Bloesch, M. A. Hoepflinger, and R. Siegwart, "Control of dynamic gaits for a quadrupedal robot," in Robotics and automation (ICRA), 2013 IEEE international conference on. IEEE, 2013, pp. 3287-3292.

[16] S. Kuindersma, R. Deits, M. Fallon, A. Valenzuela, H. Dai, F. Permenter, T. Koolen, P. Marion, and R. Tedrake, "Optimization-based locomotion planning, estimation, and control design for the atlas humanoid robot," Autonomous Robots, vol. 40, no. 3, pp. 429-455, 2016.

[17] N. Hogan, "Impedance control: An approach to manipulation, part $\mathrm{i}$ - theory," ASME Journal of Dynamic Systems, Measurement, and Control, vol. 107, pp. 1-7, 1985.

[18] C. Ott, Cartesian impedance control of redundant and flexible-joint robots. Springer, 2008.

[19] F. Aghili and C.-Y. Su, "Control of constrained robots subject to unilateral contacts and friction cone constraints," in IEEE International Conference on Robotics and Automation, 2016, pp. 2347-2352.

[20] D. Goldfarb and A. Idnani, "A numerically stable dual method for solving strictly convex quadratic programs," Mathematical programming, vol. 27, no. 1, pp. 1-33, 1983.

[21] C. Gehring, S. Coros, M. Hutler, C. D. Bellicoso, H. Heijnen, R. Diethelm, M. Bloesch, P. Fankhauser, J. Hwangbo, M. Hoepflinger, et al., "Practice makes perfect: An optimization-based approach to controlling agile motions for a quadruped robot," IEEE Robotics \& Automation Magazine, vol. 23, no. 1, pp. 34-43, 2016. 